\title{
Colitis amebiana en paciente inmunocompetente
}

\author{
S. MORÁN SÁNCHEZ, R. BAÑOS MADRID, F. GALLARDO SÁNCHEZ, \\ I. NICOLÁS DE PRADO, A. SERRANO JIMÉNEZ \\ Servicio de Digestivo. Hospital Universitario Virgen de la Arrixaca. Murcia
}

AMEBIC COLITIS IN AN IMMUNOCOMPETENT HOST

\begin{abstract}
RESUMEN
La colitis amebiana es una entidad de baja prevalencia en el mundo occidental. Su diagnóstico es infrecuente y se halla restringido a ciertos grupos de riesgo. Los hallazgos de imagen resultan ,en ocasiones, indistinguibles a los obtenidos en otras patologías tales como la Enfermedad Inflamatoria Intestinal Crónica(EIIC).

En el siguiente caso se pone de manifiesto la dificultad para realizar el diagnóstico de colitis amebiana en un sujeto inmunocompetente y sin hábitos de riesgo conocidos.
\end{abstract}

PALABRAS CLAVE: Colitis amebiana. Disenteria amebiana. Amebiasis.

\begin{abstract}
Amebic colitis is a low prevalence illness in developed countries. Its diagnosis is unfrequent and is usually made in certain groups of risk. Images studies are not useful in differencial diagnosis with other illneses as Inflamatory Bowel Disease (IBS)

Next clinical note shows the problems in the diagnosis of amebic colitis in an immunocompetent host without risk factors.
\end{abstract}

KEY WORDS: Amebic colitis. Dysentery amebic. Amebiasis.

Morán Sánchez S, Baños Madrid R, Gallardo Sánchez F, Nicolás de Prado I, Serrano Jiménez A. Colitis amebiana en paciente inmunocompetente. An Med Interna (Madrid) 2004; 21: 238-240.

\section{INTRODUCCIÓN}

La colitis amebiana es una de las entidades a tener en cuenta en el diagnóstico diferencial de la enfermedad inflamatoria intestinal crónica (EIIC).Su prevalencia en áreas desarrolladas, no endémicas, es baja y restringida a ciertos grupos de riesgo (1), lo cual contribuye a un bajo índice de sospecha de la enfermedad y su confusión con otras patologías (2).

\section{CASO APORTADO}

Presentamos a continuación a un varón de raza blanca de 46 años sin antecedentes personales ni familiares de interés. No hábitos tóxicos ni de riesgo para enfermedades de transmisión sexual o alimentaria. No evidencia de inmunodeficiencia. No viajes al extranjero.

El paciente acude a nuestro centro por síndrome diarreico de patrón disentérico con marcado deterioro del estado general. Se obtienen muestras seriadas para coprocultivo y examen fresco de heces, resultando todas ellas negativas. Se practica rectosigmoidoscopia apreciando hallazgos compatibles con EIIC tipo Crohn. El análisis microscópico de las muestras obtenidas es informado como colitis crónica inflamatoria indeterminada sin poder establecer su naturaleza exacta. Con el diagnóstico de colitis inflamatoria indeterminada se inicia terapia corticoidea oral con metilprednisona a dosis de $0,75 \mathrm{mg} / \mathrm{kg}$ obteniendo una mejoría clínica parcial. En esta situación el paciente es remitido a domicilio para proseguir control de manera ambulatoria. A los 10 dias de estancia extrahospitalaria el paciente ingresa de nuevo con la misma clínica. Se realiza enema opaco que revela la existencia de una pancolitis con importante afectación mucosa puesta de manifiesto por la visualización de ulceras en botón de camisa (Fig. 1). Se realiza de nuevo exploración endoscópica con obtención de biopsias que son informadas en el mismo sentido que las anteriores. Se aumenta la dosis de terapia corticoidea hasta niveles óptimos $(1 \mathrm{mg} / \mathrm{kg})$ con una respuesta clínica parcial. Ante la refractoriedad del cuadro se plantea el inicio de terapia inmunosupresora con azatioprina a dosis de $1,5 \mathrm{mg} / \mathrm{kg} / \mathrm{dia}$, previos controles

Trabajo aceptado: 28 de noviembre de 2003

Correspondencia: S. Morán Sánchez. C/ Sierra del Oro, 8. Urb. Altorreal. 30509 Molina de Segura (Murcia). e-mail: joaqdo@yahoo.es 


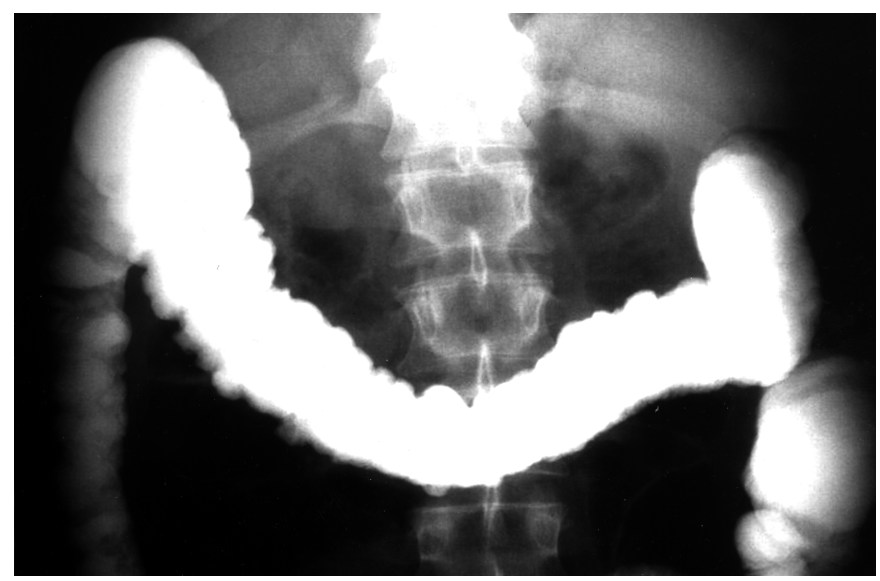

Fig. 1. Enema opaco.Imagen de afectación de mucosa colónica.

habituales. Al cabo de dos meses se mantiene una remisión clínica parcial. Se practica rectoscopia de control para obtención de biopsia. Esta muestra informa la existencia de formas amebianas con hematíes fagocitados en su interior, tanto en el exudado como invadiendo la mucosa colónica (Fig. 2). Se suspende el tratamiento inmunosupresor de inmediato y se inicia tratamiento via oral con metronidazol (500 mg/8h) con remisión total del cuadro clínico y desaparición de las lesiones endoscópicas al cabo de 10 dias. El paciente es tratado con un amebicida luminal (paramomicina $30 \mathrm{mg} / \mathrm{kg}$ ) durante 2 semanas más. Se realiza serología específica para amebas con valores que excedían 10 veces los niveles normales. Se realiza estudio de diseminación que resulta negativo. En controles sucesivos el paciente se mantiene asintomático. En el estudio de medicina preventiva no se consiguió determinar el mecanismo de transmisión. El enfermo un año después se mantiene asintomático sin tratamiento alguno.

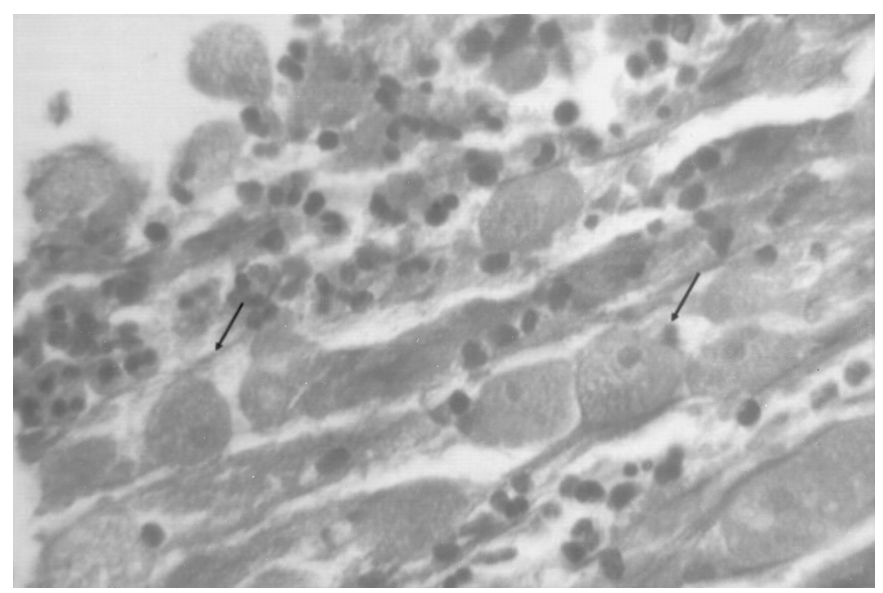

Fig. 2. Imagen microscópica de mucosa colónica. Formas amebianas (flechas negras).

\section{DISCUSIÓN}

En nuestro medio y en pacientes sin hábito de riesgo conocido es difícil sospechar la existencia de colitis amebiana. Es interesante señalar que en la flora colónica existen multitud de protozoos que no ejercen ningún efecto patogénico, y que pueden ser detectados en los análisis de heces de pacientes asintomáticos $(3,4)$. Su hallazgo, pues no constituye una indicación para tratamiento ya que forman parte de la flora comensal del individuo. Su detección no plantea problemas diagnósticos ya que morfológicamente se diferencian de la forma infectiva, Entamoeba Hystolitica, que es la que produce la enfermedad. Existe un tipo de ameba, Entamoeba Dispar, que resulta indistinguible morfológicamente de la forma infectiva (5). Hasta hace pocos años estas dos amebas se creían el mismo organismo; actualmente se consideran dos especies diferenciadas con un comportamiento radicalmente distinto. La $E$. Dispar no ejerce ningún efecto patológico en el huésped. Este hecho ha modificado de forma considerable el conocimiento que teníamos hasta la fecha de la epidemiología de la enfermedad. Así tenemos que la estimación aproximada de número de portadores a nivel mundial era de unos 500 millones de personas, actualmente se cree que sólo el $10 \%$ (unos 50 millones) de estos portadores lo son realmente de la forma infectiva, la E. Hystolitica $(6,7)$. La mayor parte de los portadores de E. Hystolitica son asintomáticos, sólo el $10 \%$ presentará algún tipo de sintomatología clínica. En áreas endémicas la diferenciación entre ambas especies plantea graves problemas de salud pública, y aunque se han desarrollado diversas técnicas de diagnóstico $(8,9)$, tales como ELISA (TechLab, Blacksburg, VA) para la detección de antígenos en heces o técnicas de diagnóstico molecular (PCR), su uso rutinario no se ha extendido en la práctica clínica diaria. La mayor parte de las áreas endémicas se hallan en países en vías de desarrollo que no son capaces de asumir el coste de estas técnicas diagnósticas

La infección por E. Hystolitica genera una respuesta inmunitaria inmediata hasta en un $90 \%$ de los pacientes. Los anticuerpos pueden ser detectados desde la primera semana de infección y persistir durante varios años (10). Un resultado negativo en la serología en estos pacientes nos ayuda a excluir la enfermedad, pero su positividad no es tan útil ya que no es capaz de diferenciarnos entre infección aguda o antigua exposición al parásito. En nuestro medio, dada la baja prevalencia de la infección un resultado positivo sería más definitorio de infección aguda que de antiguo contacto (11). La infección por E. Dispar no produce una respuesta inmunitaria. El uso de una serología específica para amebas en nuestro medio sería por tanto una herramienta útil en el diagnóstico de infección aguda amebiana. El uso de otras técnicas diagnósticas, tales como la endoscopia no ofrece avances respecto a las técnicas comentadas anteriormente. La imagen endoscópica resulta en ocasiones indistinguible a la obtenida en la EIIC por lo que no resulta útil en el diagnóstico diferencial con otras patologías que afecten al colon (12). El interés de los métodos endoscópicos reside principalmente en la capacidad para la obtención de biopsias. La biopsia obtenida de las lesiones endoscópicas ha de realizarse preferentemente en el borde de la úlcera ya que es de donde se obtiene mayor rentabilidad diagnóstica. La sensibilidad diagnóstica del examen en heces o en biopsia endoscópica es alta (80-95\%), pero el carácter acíclico y su irregular distribución pueden enmascarar su detección.

Uno de los factores predisponentes para el desarrollo de una amebiasis es la inmunodeficiencia del huésped, el paciente fue sometido a terapia inmunosupresora durante tres meses por lo que se planteó la posibilidad de que la infección fuera consecuencia del tratamiento y no la causa primaria de la coli- 
tis. Este planteamiento se desechó por la excelente respuesta a tratamiento empírico y porque el análisis "a posteriori” de las biopsias endoscópicas reveló la existencia de formas amebianas que habían pasado desapercibidas o creído integrantes de la flora saprófita del colon. La causa del retardo en el diagnóstico en este paciente es atribuible al bajo índice de sospecha de la enfermedad en un sujeto inmunocompetente en un medio no endémico. En el protocolo diagnóstico de la EIIC, entidad más prevalente en nuestro medio se realizan estudios

\section{Bibliografía}

1. Weinke T, Friedrich-Janicke B, Hopp P, Janitschke K. Prevalence and clinical importance of Entamoeba histolytica in two high-risk groups: Travelers returning from the tropics and male homosexuals. J Infect Dis 1990; 161: 1029.

2. Walsh JA. Problems in recognition and diagnosis of amebiasis: estimation of the global magnitude of morbidity and mortality. Rev Infect Dis 1986; 8: 228-38.

3. Petri WA Jr, Singh U. Diagnosis and management of amebiasis. Clin Infect Dis 1999; 29: 1117-25.

4. Aucott JN, Ravdin JI. Amebiasis and "nonpathogenic" intestinal protozoa. Infect Dis Clin North Am 1993; 7: 467.

5. Jackson TF. Entamoeba histolytica and Entamoeba dispar are distinct species; clinical, epidemiological and serological evidence. Int J Parasitol 1998; 28: 181-6.

6. Petri WA Jr, Haque R, Lyerly D, Vines RR. Estimating the impact of amebiasis on health. Parasitol Today 2000; 16: 320-1.

7. World Health Organization. Amoebiasis. Wkly Epidemiol Rec 1997; 72: $97-100$. para descartar otras etiologías; la colitis amebiana sería recomendable incluirla en el protocolo diagnóstico por las importantes connotaciones terapeúticas que conlleva (13-15). En los próximos años, con el creciente flujo migratorio se espera un aumento en la incidencia de la enfermedad, y una modificación en los factores predisponentes de la misma. Dadas las condiciones higiénico-sanitarias en nuestro medio no es probable que el aumento sea muy importante ni se establezcan áreas endémicas.
8. Haque R, Ali IKM, Akther S, Petri WA Jr. Comparison of PCR, isoenzyme analysis, and antigen detection for diagnosis of Entamoeba histolytica infection. J Clin Microbiol 1998; 36: 449-52.

9. Fredricks DN, Relman DA.Application of polymerase chain reaction to the diagnosis of infectious diseases. Clin Infect Dis 1999; 29: 475-86.

10. Krupp IM. Antibody response in intestinal and extraintestinal amebiasis. Am J Trop Med Hyg 1970; 19: 57-62.

11. Haque R, Ali IKM, Petri WA Jr. Prevalence and immune response to Entamoeba histolytica infection in preschool children in Bangladesh. Am J Trop Med Hyg 1999; 60: 1031-4.

12. Blumencranz H, Kasen L, Romeu J, Waye JD, LeLeiko NS. The role of endoscopy in suspected amebiasis.Am J Gastroenterol 1983; 78: 15-8.

13. Chan KL, Sung JY, Hsu R, Liew CT. The association of the amoebic colitis and chronic ulcerative colitis. Singapore Med J 1995; 36: 303-5.

14. Larsson PA, Olling S, Darle N. Amebic colitis presenting as acute inflammatory bowel disease.Case report. Eur J Surg 1991; 157: 553-5.

15. Li E, Stanley SL. Amebiasis. Gastroenterol Clin North Am 1996; 25: 471. 